\title{
Footwear Print Retrieval System for Real Crime Scene Marks
}

\author{
Yi Tang, Sargur N. Srihari, Harish Kasiviswanathan and Jason J. Corso \\ Center of Excellence for Document Analysis and Recognition (CEDAR) \\ University at Buffalo, The State University of New York \\ Amherst, New York 14228, U.S.A \\ \{yitang, srihari, harishka, jcorso\}@buffalo.edu \\ http://www. cedar. buffalo.edu
}

\begin{abstract}
Footwear impression evidence has been gaining increasing importance in forensic investigation. The most challenging task for a forensic examiner is to work with highly degraded footwear marks and match them to the most similar footwear print available in the database. Retrieval process from a large database can be made significantly faster if the database footwear prints are clustered beforehand. In this paper we propose a footwear print retrieval system which uses the fundamental shapes in shoes like lines, circles and ellipses as features and retrieves the most similar print from a clustered database. Prints in the database are clustered based on outsole patterns. Each footwear print pattern is characterized by the combination of shape features and represented by an Attributed Relational Graph. Similarity between prints is computed using Footwear Print Distance. The proposed system is invariant to distortions like scale, rotation, translation and works well with the partial prints, color prints and crime scene marks.
\end{abstract}

Keywords: Footwear Impression Evidence, ARG, Footwear Print Distance, Hough transform, Content-based Image Retrieval

\section{Introduction}

Footwear marks are often found in crime scenes and can serve as a clue to link two crimes. At present there is no reliable and fully automated footwear print retrieval system to assist the forensic examiners. Therefore the foot wear marks are manually matched against the local/national database to determine the brand and model of the most similar print. Existing footwear print retrieval systems works well only with clear prints but the crime scene marks are highly degraded and partial. Most of the present systems [1] fail with crime scene marks because they use features that are hard be captured from the crime scene marks.

Retrieval process can be made faster if prints in the database are grouped into clusters based on similar patterns. Clustering footwear prints is an uphill task because of the difficulty in the computation of the similarity matrix. Hence, we cluster them by retrieving the most similar prints for each of the recurring 
patterns in footwear prints. The proposed fully automated footwear print retrieval system (shown in Figure1) is invariant to affine transformation of prints and avoids the computation of similarity matrix.

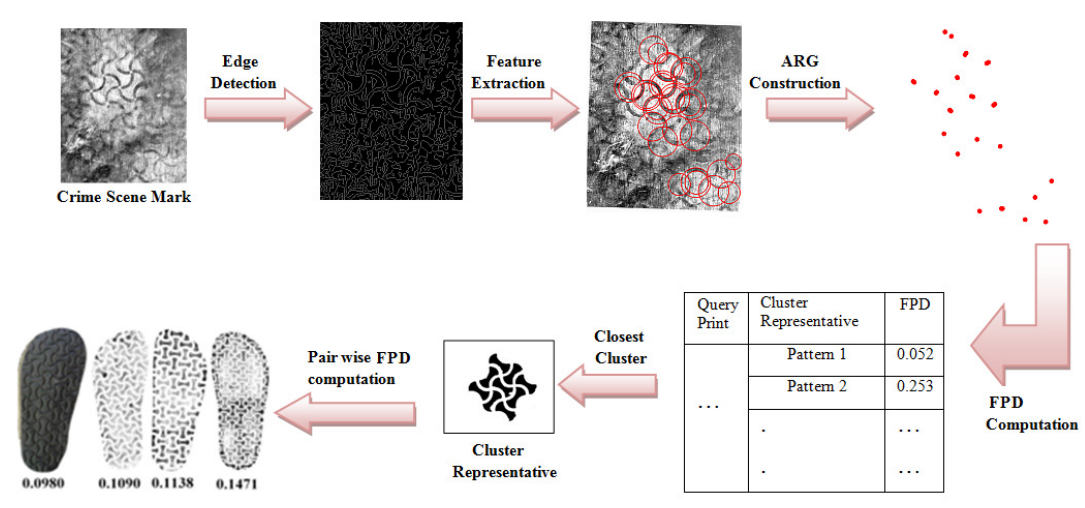

Fig. 1: System Flow Diagram of our footwear print retrieval system.

Most of the existing shoe print classification systems are semi-automatic [1] , such as SICAR, SoleMate etc. De Chazal (2005) et al. [2] proposed a fully automated shoe print classification system which uses power spectral density (PSD) of the print as a pattern descriptor. Zhang and Allinson (2005) [3] proposed an automated shoe print retrieval system in which 1-D Discrete Fourier Transform on the normalized edge direction histogram is used as a feature of the print. Gueham (2008) et al. [6] evaluated the performance of 'Optimum Trade-off Synthetic Discriminant Function (OTSDF)' filter and unconstrained OTSDF filter in the automatic classification of partial shoeprints. Dardi (2009) et al. [7] described a texture based retrieval system for shoeprints. Mahalanobis map is used to capture the texture and then matched using correlation coefficient. Though fully automated footwear print retrieval system exists in literature, most of them is tested only with synthetic prints and clean prints. No one has reported satisfying performance with real crime scene marks.

\section{Feature Extraction}

Features such as color, texture and shape [8] can be used to distinguish the images. Color features are missing in crime scene marks and texture features are hard to be captured but shape features can be easily captured. Shapes are the most durable and reliable features of the outsole that can resist various wears and are well preserved over a long period of time. Hence, we chose shape as features to classify footwear prints.

Based on visual inspection of 5034 prints, we found that $91.8 \%$ of the prints can be represented using three basic shapes: straight line segments, circles/arcs and ellipses. Based on these shapes, footwear prints can be classified into 8 types: 
Piecewise Lines, Only Circles/Arcs, Only Ellipses, Circles \& Ellipses, Lines \& Circles, Lines \& Ellipses, Lines, Circles \& Ellipses and Only Texture. Shapes other than circles and ellipses are approximated by piecewise lines. Combinations of these shapes is used to identify the pattern of the shoeprint. Distribution of fundamental shapes in footwear prints are shown in Table 1. Eight types of footwear prints are shown in Figure 2.

Table1: Fundamental shapes in footwear prints

\begin{tabular}{|c|c|}
\hline Fundamental Shapes & Number of Prints \\
\hline Piecewise Lines & 3397 \\
\hline Lines \& Circles & 812 \\
\hline Lines \& Ellipses & 285 \\
\hline Only Circles/Arcs & 73 \\
\hline Lines, Circles \& Ellipses & 37 \\
\hline Only Ellipses & 15 \\
\hline Circles \& Ellipses & 5 \\
\hline Only Texture & 410 \\
\hline \multicolumn{2}{|c|}{ Total - 5034 prints } \\
\hline
\end{tabular}
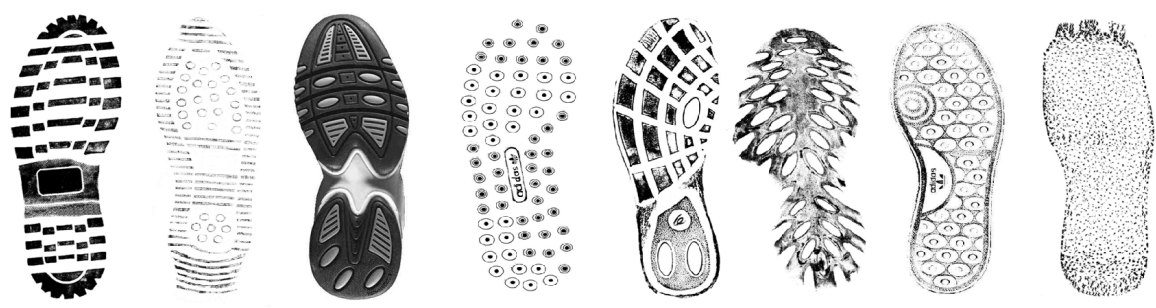

Fig. 2: Eight types of Footwear prints: From left to right (a) Piecewise Lines. (b) Lines \& Circles. (c) Lines \& Ellipses (d) Only Circles/Arcs. (e) Lines, Circles \& Ellipses. (f) Only Ellipses. (g) Lines, Circles \& Ellipses. (h) Circles \& Ellipses. (i) Only Texture.

Morphological operations (MO) such as dilation and erosion are applied to make the interior region of prints uniform and then extract the boundary using Canny edge[9] detector(ED). The result for a sample print is shown in Figure 3.

\subsection{Line Detection and Circle Detection}

Most footwear prints have complex geometric structures. The number of line segments in a print is 200-300 on average. Each group of collinear points forms a peak in accumulator. Detecting all the true peaks while suppressing spurious ones is difficult. In addition, short line segments are easily missed, which may be useful for discriminating similar prints. So Standard Hough Transform (SHT) [10] cannot be applied directly on footwear prints. Thus, we propose Iterative 

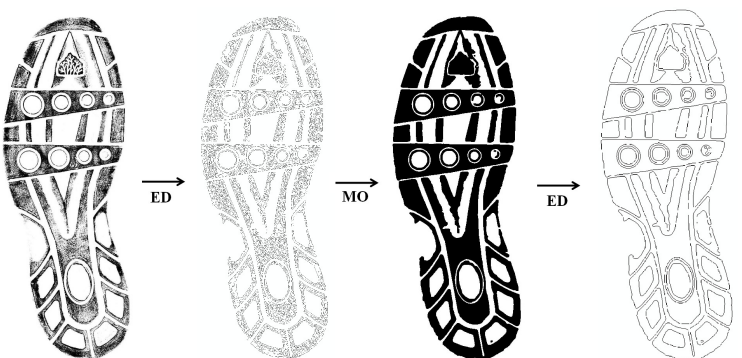

Fig. 3: Edge Detection after dilation and erosion.

Straight-line Hough Transform (ISHT). First, connected components are labeled in the edge image. For each component, Hough transform is applied and peaks are detected. When a peak is identified and the line segments are extracted, the pixels contributing to those line segments are eliminated from the edge image, and an updated accumulator is obtained by applying SHT on the modified edge image. The algorithm is summarized in Figure 4 and results of the two algorithms on two sample prints are shown in Figure 5.

Circles and arcs are detected using SHT and validated using gradient orientation. Spatial relationship constraints are used to eliminate spurious circles.

\subsection{Ellipse Detection}

In a Cartesian plane, an ellipse is described by its centre $(p, q)$, length of the semi-major axis $a$, length of the semi-minor axis $b$ and the angle $\theta$ between the major axis and the $x$-axis. Hence the five parameters $(p, q, a, b, \theta)$ are required to uniquely describe an ellipse. These five parameters demand a five dimensional accumulator which is computationally expensive but Randomized Hough transform (RHT) [11] for ellipse detection is computationally advantageous.

In case of ellipse detection in footwear prints, RHT cannot be used directly. This is because there are around 50,000 foreground pixels in the edge image of a print of typical size $600 \times 800$ and picking three pixels from them in random will never narrow down to the right ellipse. Hence, we propose a modified RHT (MRHT) that incorporates ideas like decomposition of footwear prints into connected components, elimination of unwanted components using eccentricity, smart selection of three points based on the standard deviation of gradient orientation at each pixel and elimination of spurious ellipses by comparing the tangent of edge direction and the analytical derivative. MRHT is shown as Algorithm 2. Sample Results of the feature extraction are shown in Figure 6.

\section{Attributed Relational Graph (ARG)}

After feature extraction, the footwear print has been decomposed in to a set of primitives. An Attributed Relational Graph (ARG) [12], is constructed for every footwear print to obtain a structural representation of the extracted features. An ARG is a 3 -tuple $(V, E, A)$ where $V$ is the set of nodes, $E$ is the set of edges and 


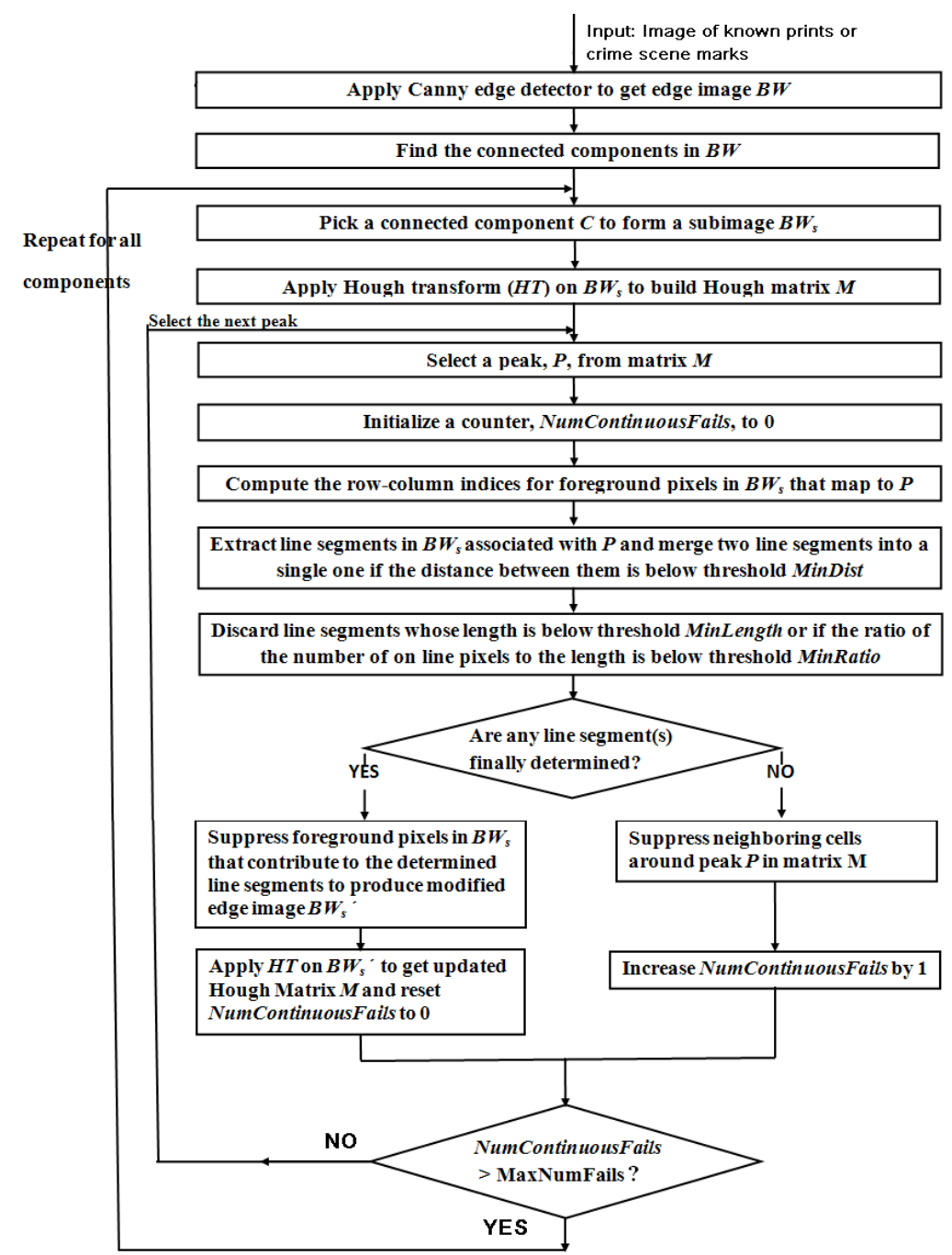

Fig. 4: Algorithm 1: Iterative Straight-line Hough Transform (ISHT)

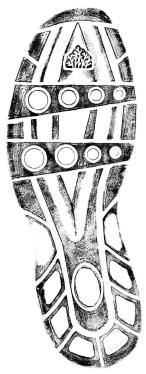

(a) Print

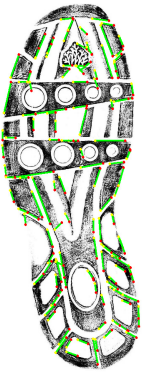

(b) SHT

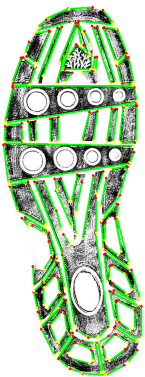

(c) ISHT

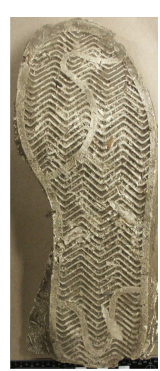

(d) Print

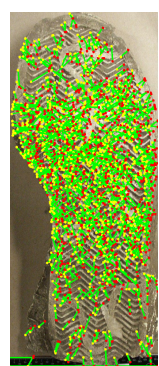

(e) SHT

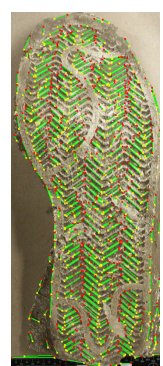

(f) ISHT

Fig. 5: Comparison of the results of SHT and ISHT on sample prints 


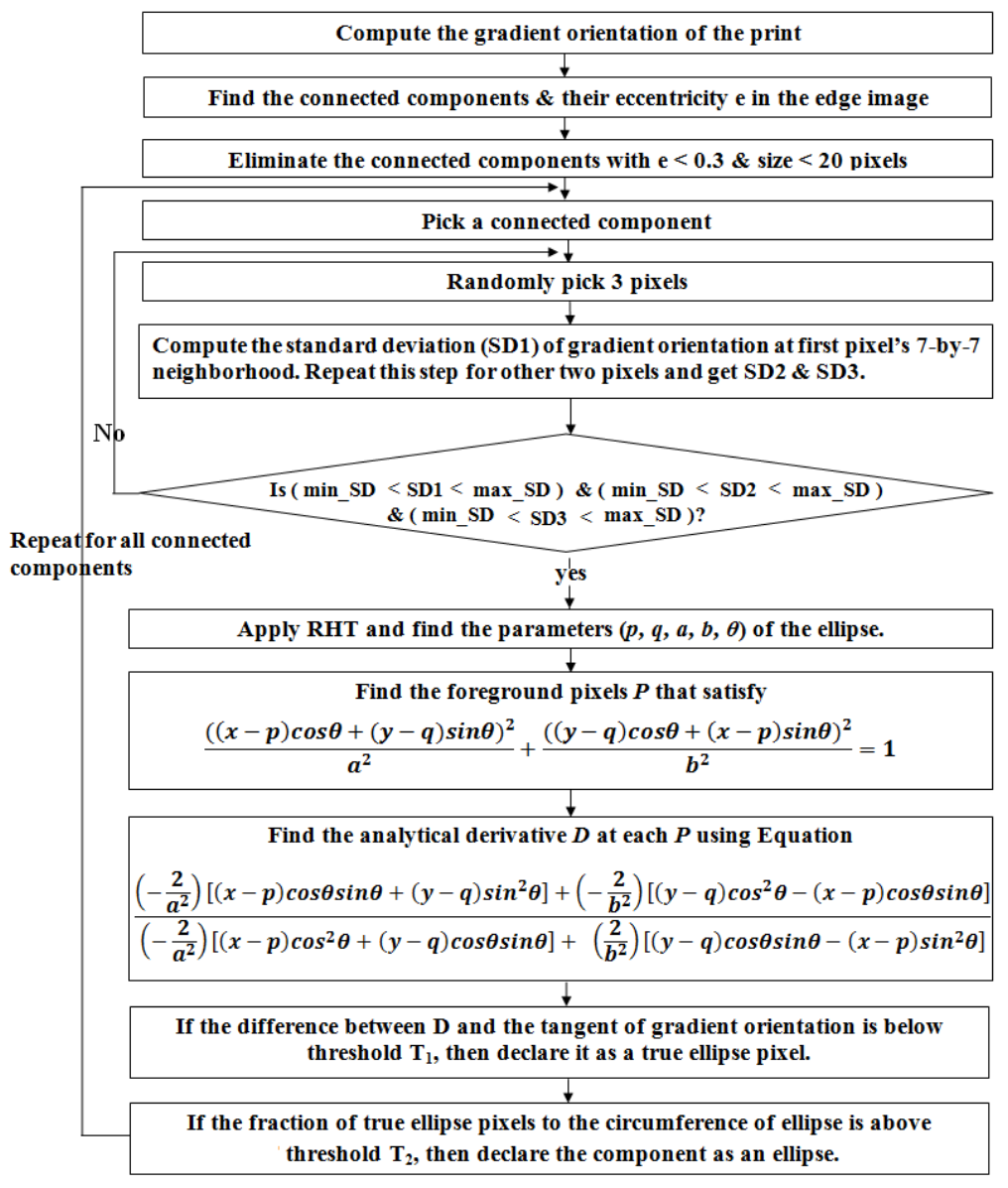

Algorithm 2: MRHT
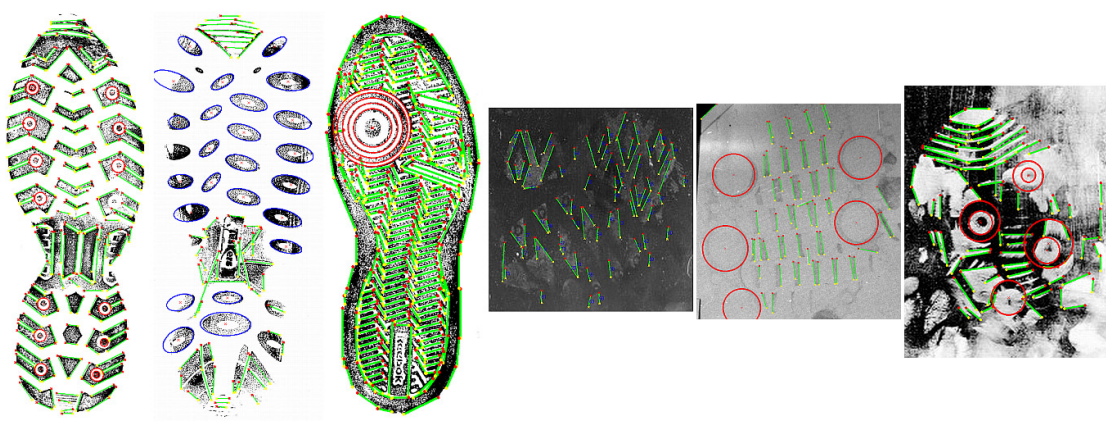

Fig. 6: Detected lines, circles and ellipses are shown for 3 known prints on the left and 3 crime scene marks on the right. 
$A$ is the set of attributes. Each edge describes the spatial relationship between nodes. The attributes include node and edge attributes.

There are three types of nodes (lines, circles and ellipses) and nine types of edges (line-to-line, line-to-circle, line-to-ellipse, circle-to-line, circle-to circle, circle-to-ellipse, ellipse-to-line, ellipse-to-circle and ellipse-to-ellipse) in this representation, which are denoted as L2L, L2C, L2E, C2L, C2C, C2E, E2L, E2C, $\mathrm{E} 2 \mathrm{E}$ edges. To tackle the case of nodes being missing or incorrectly detected due to noise, occlusion and incompleteness, a fully-connected directed graph is adopted. This means that there is a directed edge from each node to all other nodes. To distinguish one node from the other and one pair of nodes from another pair, node and edge attributes have been carefully designed to describe the spatial relationship between nodes in terms of distance, relative position, relative dimension and orientation, and are tabulated in Table 2. All these attributes have been normalized within the range $[0,1]$. This description is invariant to scale, rotation, translation and insensitive to noise and degradations. Figure 7 describes the two cases of relative position for L2L, L2C and C2E. Sample ARG for a footwear print is shown in Figure 8.
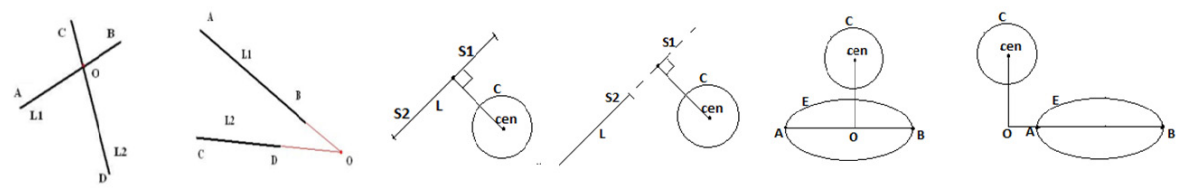

Fig. 7: Relative Position Definition for L2L, L2C and C2E.

\section{Footwear Print Distance (FPD)}

The quality of clusters depends highly on the similarity measure used by the clustering algorithm so the similarity measure has to be accurate and robust. A distance metric, called the Earth Mover's Distance (EMD) [13] has been proposed and investigated for content-based image retrieval.

We propose Footwear Print Distance (FPD), which is derived from EMD, to compute the similarity between footwear prints. Let $F P=(V, E, A, W, N)$ be a footwear print with $N$ nodes, where $V=\left\{V_{i}\right\}_{i=1}^{N}, E=V \times V, A=A_{N} \cup A_{E}$, $A_{N}=\bigcup_{i=1}^{N} A_{N i}, A_{E}=\bigcup_{i=1}^{N} \bigcup_{j=1}^{N} A_{E i j}, W=\left\{W_{i}\right\}_{i=1}^{N} . A_{N}, A_{E}$ and $W$ denote node attribute, edge attribute and weights respectively. Let $F P_{1}=$ $\left(V_{1}, E_{1}, A_{1}, W_{1}, N_{1}\right)$ be the first print with $N_{1}$ nodes; $F P_{2}=\left(V_{2}, E_{2}, A_{2}, W_{2}, N_{2}\right)$ the second print with $N_{2}$ nodes; and $C=\left[c_{i j}\right]$ be the unit cost matrix where $c_{i j}$ is the unit matching cost between $V_{1 i} \in V_{1}$ and $V_{2 j} \in V_{2}$, which is defined as follows. If $V_{1 i}$ is a line and $V_{2 j}$ is a circle/ellipse or vice-versa, then $c_{i j}=1$ else $c_{i j}$ is computed as an EMD [13] in which the distance between any two nodes is defined as a weighted Euclidean distance between their node and edge attributes. Each node is assigned equal weight to enforce a one-to-one correspondence between nodes, i.e. $W_{1 i}=W_{2 j}=\frac{1}{\max \left(N_{1}, N_{2}\right)}, 1 \leq i \leq N_{1}, 1 \leq j \leq N_{2}$. 
Table 2: Node \& Edge Attributes

\begin{tabular}{|c|c|c|}
\hline Att & Definition & Normalization \\
\hline $\mathrm{N}-\alpha$ & $\frac{\operatorname{abs}(L 1 . \theta-L 2 . \theta)}{180}$ & - \\
\hline$N-r s$ & $\frac{\text { L1.len }}{\text { L1.len+L2.len }}$ & - \\
\hline$r d$ & $\frac{\operatorname{dist}(L 1 . m, L 2 . m)}{L 1 . \operatorname{len}+L 2 . \operatorname{len}}$ & $2(\sigma(r d)-0.5)$ \\
\hline$p d$ & $\frac{\operatorname{dist}(L 1 . m, L 2)}{L 1 . \text { len }+ \text { L2.len }}$ & $2(\sigma(p d)-0.5)$ \\
\hline$r p_{1}$ & $\frac{\min (O A, O B)}{\max (O A, O B)}$ & $\frac{r p 1+1}{2}$ \\
\hline$r p_{2}$ & $\frac{\min (|O A|,|O B|)}{\max (|O A|,|O B|)}$ & $\frac{r p 2+1}{2}$ \\
\hline \multicolumn{3}{|c|}{ L2E } \\
\hline$e$ & E.e & - \\
\hline$N-r s$ & $\frac{\text { L.len }}{\text { L.len }+E . E R}$ & - \\
\hline$r d$ & $\frac{\operatorname{dist}(\text { L.m,E.cen })}{\text { L.len }+ \text { E.ER }}$ & $2(\sigma(r d)-0.5)$ \\
\hline$r p 1$ & $\frac{\min (O A, O B)}{\max (O A, O B)}$ & $\frac{r p 1+1}{2}$ \\
\hline$r p 2$ & $-\frac{\min (|O A|,|O B|)}{\max (|O A|,|O B|)}$ & $\frac{r p 2+1}{2}$ \\
\hline N-ro & $\frac{a b s(L \cdot \theta-E . \theta)}{180}$ & - \\
\hline \multicolumn{3}{|c|}{$\mathrm{C} 2 \mathrm{E}$} \\
\hline$e$ & E.e & - \\
\hline$N-r s$ & $\frac{C \cdot r}{C \cdot r+E \cdot E R}$ & - \\
\hline$r d$ & $\frac{\text { dist (C.cen, E.cen })}{\text { C.r+E.ER }}$ & $2(\sigma(r d)-0.5)$ \\
\hline$r p$ & $\frac{\min (O A, O B)}{\max (O A, O B)}$ & $\frac{r p+1}{2}$ \\
\hline
\end{tabular}

\begin{tabular}{|c|c|c|}
\hline \multicolumn{3}{|c|}{$\mathrm{C} 2 \mathrm{C}$} \\
\hline Att & Definition & Normalization \\
\hline \multirow{2}{*}{ N-rs } & C1.r & \multirow[b]{2}{*}{ - } \\
\hline & $\overline{C 1 . r+C 2 . r}$ & \\
\hline \multirow{2}{*}{$r d_{1}$} & dist (C1.cen,C2.cen) & $r d 1$ \\
\hline & $C 1 . r+C 2 . r$ & $r d 1+10 / r d 1$ \\
\hline \multirow{2}{*}{$r d_{2}$} & dist (C1.cen,C2.cen $)$ & $r d 2$ \\
\hline & $\max \left(|C 1 . r-C 2 . r|, 10^{-3}\right)$ & $r d 2+10 / r d 2$ \\
\hline \multicolumn{3}{|c|}{ L2C } \\
\hline \multirow{2}{*}{$N-r s$} & L.len & \multirow[b]{2}{*}{ - } \\
\hline & $\overline{C . r+\text { L.len }}$ & \\
\hline \multirow[b]{2}{*}{$r d$} & $\operatorname{dist}($ C.cen, $L)$ & $r d$ \\
\hline & C.r & $\overline{r d+10 / r d}$ \\
\hline \multirow{2}{*}{$r p$} & $\min (S 1, S 2)$ & $r p+1$ \\
\hline & $\overline{\max (S 1, S 2)}$ & 2 \\
\hline
\end{tabular}

C2L

\begin{tabular}{|c|c|c|}
\hline$N-r s$ & $\frac{\text { C.r }}{\text { C.r+L.len }}$ & - \\
\hline$r d$ & $\frac{\text { dist }(\text { C.cen, } L)}{\text { C.r }}$ & $\frac{r d}{r d+10 / r d}$ \\
\hline
\end{tabular}

Symbols Used \& its Definition

$L$ : Line segment $c:$ Circle

E: Ellipse cen: Centre Dist: Euclidean distance

$r s$ : Relative size

$r$ : Radius

Len: Length $r p$ : Relative position e: Orientation E: eccentricity Att: attributes

$E R: \sqrt{a * b}$ $N$ : Normalized

\begin{tabular}{|c|c|c|}
\hline \multirow{2}{*}{$e_{-}$ratio } & $\frac{\text { E1.e }}{\text { E1.e +E2.e }}$ & - \\
\hline$f(4 e)$ & $\frac{\text { E1.e-E2.e }+1}{2}$ & - \\
\hline$r d$ & $\frac{\text { dist(E1.cen,E2.cen) }}{\text { E1.ER+E2.ER }}$ & $\frac{r d}{r d+10 / r d}$ \\
\hline$N-r s$ & $\frac{\text { E1.ER }}{\text { E1.ER+E2.ER }}$ & - \\
\hline$N-r o$ & $\frac{a b s(E 1 . \theta-E 2 . \theta)}{90}$ & - \\
\hline$r p$ & $\begin{array}{c}\text { rp(E1.major-axis, } \\
\text { E2.major-axis) }\end{array}$ & $\frac{r p+1}{2}$ \\
\hline
\end{tabular}

mid: mid-point $\sigma(x): \frac{1}{1+e^{-x}}$ pd: perpendicular distance

Ellipse:

a: Semi-major axis. b: Semi-minor axis. $p, q$ : centre

max: maximum

| |: absolute

abs: absolute

\section{Node Attributes}

\begin{tabular}{|c|c|c|c|c|c|c|c|c|}
\hline Node & Attributes & Definition & Node & Attributes & Definition & Node & Attributes & Definition \\
\hline Circle & Completeness & $\begin{array}{l}\text { Standard deviation of the angle that } \\
\text { on-circle pixels make w.r.t. center }\end{array}$ & Circle & Quality & $\frac{\text { Number of pixels on circle }}{\text { Circumference of circle }}$ & Ellipse & Eccentricity & $1-\frac{b^{2}}{a^{2}}$ \\
\hline
\end{tabular}
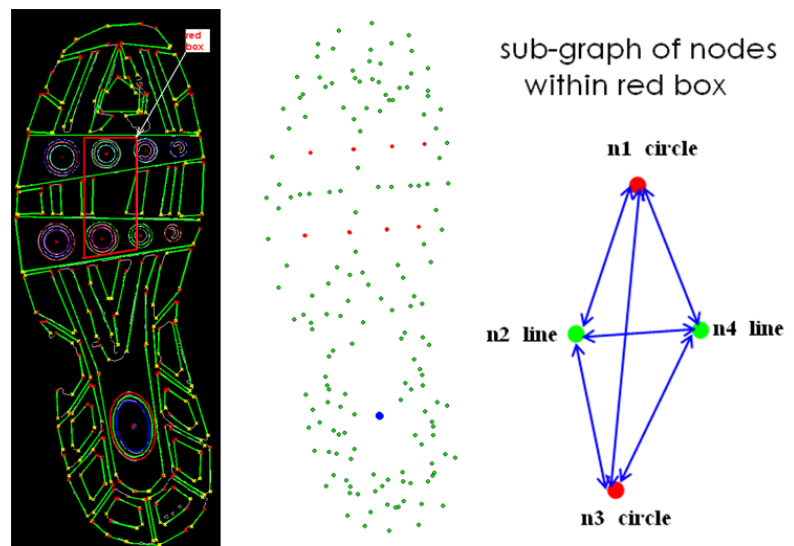

Fig. 8: Extracted features of a shoeprint shown in Fig. 5(a), its ARG and sub-graph of nodes within red box. The ARG is fully connected and its edges are omitted for clarity. 
The goal is to calculate a node correspondence matrix $M=\left[m_{i j}\right]$, where $m_{i j}$ denotes the amount of weight transferred from $V_{1 i}$ to $V_{2 j}$, which minimizes the total matching cost $\operatorname{Cost}\left(F P_{1}, F P_{2}, M\right)=\sum_{i=1}^{N_{1}} \sum_{j=1}^{N_{2}} m_{i j} * c_{i j}$, subject to the following constraints:

$$
\begin{gathered}
m_{i j} \geq 0, \quad 1 \leq i \leq N_{1}, 1 \leq j \leq N_{2} \\
\sum_{j=1}^{N_{2}} m_{i j} \leq W_{1 i}, 1 \leq i \leq N_{1} \\
\sum_{i=1}^{N_{1}} m_{i j} \leq W_{2 j}, 1 \leq j \leq N_{2} \\
\sum_{i=1}^{N_{1}} \sum_{j=1}^{N_{2}} m_{i j}=\min \left(\sum_{i=1}^{N_{1}} W_{1 i}, \sum_{j=1}^{N_{2}} W_{2 j}\right)
\end{gathered}
$$

Once the correspondence matrix is found, the FPD is defined as the overall cost normalized by the sum of all the weights transferred from $F P_{1}$ to $F P_{2}$.

$$
F P D\left(F P_{1}, F P_{2}\right)=\frac{\sum_{i=1}^{N_{1}} \sum_{j=1}^{N_{2}} m_{i j} * c_{i j}}{\sum_{i=1}^{N_{1}} \sum_{j=1}^{N_{2}} m_{i j}}
$$

\section{Clustering Footwear Prints}

Clustering algorithms can be generally divided into partition-based, densitybased and hierarchical based methods [14]. Existing clustering algorithms like K-means, Hierarchical Clustering, and Expectation Maximization requires similarity matrix consisting of pair-wise distance between every footwear prints in the dataset. Building similarity matrix for a large dataset is computationally expensive. Hence, to cluster the entire dataset we propose using recurring patterns as fixed cluster center.

Recurring patterns (shown in Fig.9(a)) such as wavy pattern, concentric circles etc. are common in footwear prints and can be used to represent a group of similar prints. These patterns are simple in structure and graphs constructed from them have less number of nodes. Hence, recurring patterns can be used as query to fetch all similar prints from the database. This drastically reduced the computation and did not require similarity matrix. Though this clustering method requires domain knowledge to determine the recurring patterns, it avoids the problems of deciding the number of clusters beforehand (unlike K-means).

From visual inspection, 25 recurring patterns (shown in Figure9(a)) were determined and used as cluster representatives. FPD between each database print and every cluster representative was calculated. Then each print was assigned to the nearest representative, for which the FPD is below threshold $T$. If FPD between a print and cluster representatives are greater than $T$, then the print remains as a single cluster. In our experiments, $\mathrm{T}$ was set to 0.15 . 1410 shoeprints were fitted in to these 25 clusters. Remaining 1250 prints were so unique that each of them was a cluster by itself. Sample clusters are shown in Figure 9(b). 


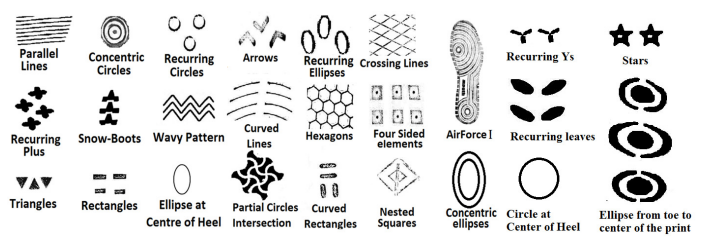

(a)

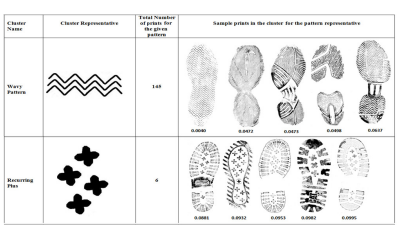

(b)

Fig. 9: (a) Cluster Representatives. (b) Sample Clusters.

\section{Experiments and Results}

A database of 2660 known prints and 100 crime scene marks was used for experiments. Each known print has the meta data such as brand and model of the footwear, which is useful for linking a suspect's footwear to a crime scene mark. In the clustered database, crime scene mark was first matched against every cluster representative to find the closest cluster, and then matched against each print in that cluster and the top $n$ matches are retrieved. Retrieval results for 4 sample marks are shown in Figure 10.
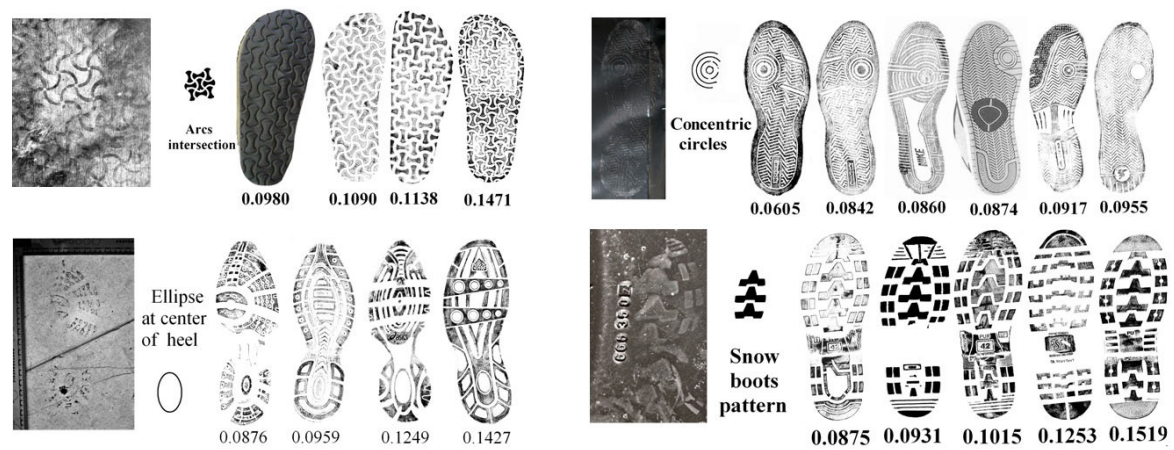

Fig. 10: Crime Scene marks, their closest cluster and closest matches from the cluster.

The system's performance has been evaluated using 100 real crime scene marks and 1400 degraded prints, which were obtained by applying combinations of affine transformation, partitioning and noise degradation on certain database prints. Cumulative Match Characteristic (CMC) [2] was chosen as the performance metric because it can answer the question "What is the probability of a match in the first $n$ percent of the sorted database?". The probability of a match (cumulative match score) is estimated by the proportion of times a matching footwear print appears in the first $n$ percent of the sorted database. SIFT [15] being a robust image matching algorithm, we compared our system with SIFT. The CMC curve of our system before clustering and of SIFT are shown in Fig. 11. CMC curve remains the same before and after clustering but clustering makes significant improvement in the retrieval speed, which is shown in Table 3.

Dardil et al. [7] are the only one who have worked with real crime scene marks. They have reported that $73 \%$ of the real crime scene marks were found 


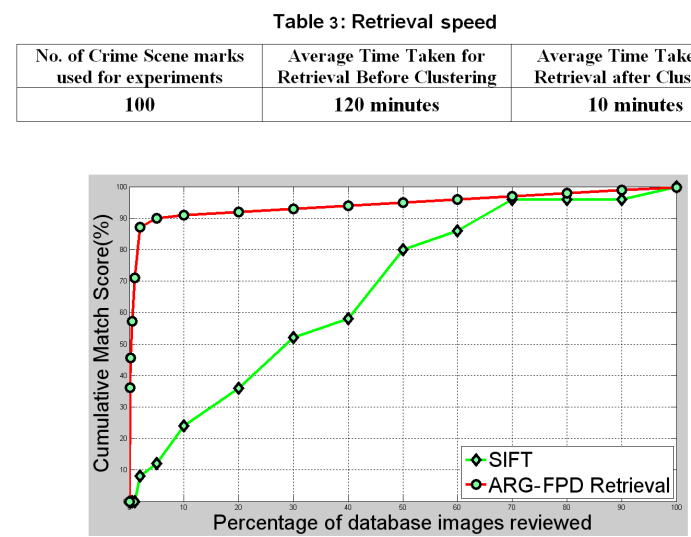

Fig. 11: CMC of Our System and SIFT on Retrieval of Crime Scene Marks.

in the top $10 \%$ of database prints but they have tested their system with only 87 known prints and 30 real crime scene marks. From CMC of our system, it is clear that, $91 \%$ of matching prints for the crime scene marks were found in the top $10 \%$ of the database prints. Table 4 compares our work with the state of the art techniques and our system performed better than the others for full prints, partial prints and real crime scene marks. Accuracy of $100 \%$ with degraded full and partial prints demonstrates the invariance of our system to affine transformation and robustness against noise and degradations.

Table 4: Comparison of ARG-FPD with the State-of-the-art

\begin{tabular}{|c|c|c|c|c|c|c|c|c|c|}
\hline \multirow{2}{*}{$\begin{array}{c}\text { State-of- } \\
\text { the-art }\end{array}$} & \multicolumn{6}{|c|}{$\begin{array}{l}\text { Query from the leaning set/similar to one in learning set } \\
\text { Full print } \\
\text { Partial print }\end{array}$} & \multirow{2}{*}{$\begin{array}{l}\text { Experiments } \\
\text { using Real Crime } \\
\text { Scene marks }\end{array}$} & \multirow[b]{2}{*}{ Shortcoming } & \multirow[b]{2}{*}{ Dataset } \\
\hline & CMS at $1 \%$ & $\begin{array}{l}\text { Full print } \\
\text { CMS at } 5 \%\end{array}$ & $\begin{array}{l}\text { CMS at } \\
10 \%\end{array}$ & $\begin{array}{l}\text { CMS at } \\
1 \%\end{array}$ & $\begin{array}{l}\text { Partial prit } \\
\text { CMS at } \\
5 \%\end{array}$ & $\begin{array}{c}\text { CMS at } \\
10 \%\end{array}$ & & & \\
\hline $\begin{array}{c}\text { Chazal et al. [2] } \\
\text { (Using Power } \\
\text { Spectral Density) }\end{array}$ & 64 & 87 & 90 & 50 & 70 & 77 & - & $\begin{array}{l}\text { Lacks Scaling } \\
\text { invariance. }\end{array}$ & $\begin{array}{c}\mathbf{4 7 5} \text { prints from } \\
\text { dataset of Forensic } \\
\text { Science Laboratory, } \\
\text { Dublin, Ireland }\end{array}$ \\
\hline $\begin{array}{c}\text { Zhang et al. [3] } \\
\text { (Using Edge } \\
\text { Direction } \\
\text { Histogram) }\end{array}$ & 85.4 & 95 & 97.44 & - & - & - & - & $\begin{array}{l}\text { Not tested with } \\
\text { partial prints. }\end{array}$ & $\begin{array}{c}512 \text { prints from } \\
\text { Foster \& Freeman } \\
\text { Dataset }\end{array}$ \\
\hline $\begin{array}{c}\text { Pavlou et al. [4] } \\
\text { (Using Gradient } \\
\text { Location and } \\
\text { Orientation } \\
\text { Histogram) } \\
\end{array}$ & 86 & 90 & 93 & 85 & 90 & 92 & - & $\begin{array}{l}\text { Not tested with } \\
\text { real crime } \\
\text { scene marks }\end{array}$ & $\begin{array}{l}368 \text { prints provided } \\
\text { by Forensic Science } \\
\text { Services dataset, UK }\end{array}$ \\
\hline $\begin{array}{l}\text { Crookes et al. [5] } \\
\text { (Using Local } \\
\text { Image Features) }\end{array}$ & 100 & 100 & 100 & 100 & 100 & 100 & - & $\begin{array}{l}\text { Tested with only } \\
\text { synthesized SoCs }\end{array}$ & $\begin{array}{c}500 \text { clean prints and } \\
50 \text { degraded prints }\end{array}$ \\
\hline $\begin{array}{l}\text { Crookes et al. [5] } \\
\text { (Using phase only } \\
\text { correlation) }\end{array}$ & 100 & 100 & 100 & 100 & 100 & 100 & - & $\begin{array}{c}\text { Lacks } \\
\text { rotational } \\
\text { invariance }\end{array}$ & $\begin{array}{c}100 \text { clean prints and } \\
64 \text { synthetic scene } \\
\text { images }\end{array}$ \\
\hline $\begin{array}{l}\text { Gueham et al. [6] } \\
\text { (Using Advanced } \\
\text { Correlation } \\
\text { Filters) }\end{array}$ & - & - & - & 95.68 & - & - & - & $\begin{array}{c}\text { Tested only } \\
\text { with } 100 \text { prints }\end{array}$ & $\begin{array}{l}100 \text { prints from } \\
\text { Foster \& Freeman } \\
\text { Dataset }\end{array}$ \\
\hline \multirow{3}{*}{$\begin{array}{l}\text { Dardil et al. [7] } \\
\text { (Using texture) }\end{array}$} & \multirow{3}{*}{ - } & \multirow{3}{*}{-} & \multirow{3}{*}{ - } & \multirow{3}{*}{-} & \multirow{3}{*}{-} & \multirow{3}{*}{ - } & CMS at $10 \%$ is $73 \%$ & \multirow{3}{*}{$\begin{array}{c}\text { Tested only } \\
\text { with } 87 \text { known } \\
\text { prints and } 30 \\
\text { SoCs }\end{array}$} & \multirow{3}{*}{$\begin{array}{l}87 \text { known prints and } \\
30 \text { real crime scenes } \\
\text { from ENSFI group }\end{array}$} \\
\hline & & & & & & & CMS at $5 \%$ is $40 \%$ & & \\
\hline & & & & & & & CMS at $1 \%$ is $10 \%$ & & \\
\hline \multirow{2}{*}{$\begin{array}{l}\text { ARG-FPD } \\
\text { Approach }\end{array}$} & \multirow[b]{2}{*}{100} & \multirow[b]{2}{*}{100} & \multirow{2}{*}{100} & \multirow{2}{*}{100} & \multirow[b]{2}{*}{100} & \multirow[b]{2}{*}{100} & CMS at $10 \%$ is $91 \%$ & & \multirow{2}{*}{$\begin{array}{l}1400 \text { degraded prints, } \\
100 \text { real crime scenes } \\
\text { \& } \\
2660\end{array}$} \\
\hline & & & & & & & $\begin{array}{l}\text { CMS at } 5 \% \text { is } 90 \% \\
\text { CMS at } 1 \% \text { is } 71 \%\end{array}$ & & \\
\hline
\end{tabular}




\section{Conclusion}

The proposed Attributed Relational Graph representation of footwear prints works well with crime scene marks. Our system is invariant to affine transformation and robust against noise and various image degradations. Performance of the system clearly shows that fundamental shapes in shoes are one of the most reliable features, ARG is a robust descriptor of these features and FPD is an ideal similarity measure to match partial and degraded prints. Clustering using recurring patterns is a promising method to cluster footwear prints and it certainly enhances the retrieval speed with same accuracy.

\section{References}

1. Bouridane, A.: Imaging for Forensics and Security: From Theory to Practice. Springer, New York (2009)

2. De Chazal, P.D., Reilly, R.: Automated processing of shoeprint images based on the fourier transform for use in forensic science. In: IEEE Transaction on Pattern Analysis and Machine Intelligence, pp. 341-350. IEEE Press (2005)

3. Zhang, L., Allinson, N.: Automatic shoeprint retrieval system for use in forensic investigations. In: 5th Annual UK Workshop on Computational Intelligence (2005)

4. Pavlou, M., Allinson, N.M.: Automatic extraction and classification of footwear patterns. In: Proc. Intelligent Data Engineering and Automated Learning (2006)

5. Crookes, D., Bouridane, A., Su H., Gueham M.: Following the Footsteps of Others: Techniques for Automatic Shoeprint Classification. In: $2^{\text {nd }}$ NASA/ESA Conference on Adaptive Hardware and Systems (2007)

6. Gueham, M., Bouridane, A., Crookes, D.: Automatic classification of Partial Shoeprints using Advanced Correlation Filters for use in Forensic Science. In: International Conference on Pattern Recognition, pp. 1-4. IEEE Press (2008)

7. Dardi, F., Cervelli, F., Carrato, S.: A Texture based Shoe Retrieval System for Shoe Marks of Real Crime Scenes. In: International Conference on Image Analysis and Processing, pp. 384-393. IEEE Press (2009)

8. Rui, Y., Huang, S., Chang, S.: Image retrieval: Current techniques, promising directions, and open issues. J. Visual Communication and Image Representation. 10, 39-62 (1999)

9. Nixon, M., Aguado, A.: Feature Extraction and Image Processing. Elsevier Science, Oxford (2002)

10. Hough,P.V.C.: Method and means for recognizing complex patterns, US Patent 3069654 (1962)

11. McLaughlin, R.: Randomized Hough transform: better ellipse detection. In: IEEE TENCON-Digital Signal Processing Applications, pp. 409-414. IEEE Press (1996)

12. Sanfeliu, A., Fu, K. S.: A distance measure between attributed relational graphs for pattern recognition. In: IEEE transactions on systems, man, and cybernetics, pp. 353-362. IEEE Press (1983)

13. Rubner, Y., Tomasi, C., Guibas, L.J.: The earth movers distance as a metric for image retrieval. International Journal of Computer Vision. 40, 99-121 (2000)

14. Han, J., Kamber, M., Pei, J.: Data Mining: Concepts and Techniques. Morgan Kaufmann (2005)

15. Lowe, D.G.: Distinctive image features from scale-invariant keypoints. International Journal of Computer Vision. 40, 91-110 (2004) 